Bull. Korean Math. Soc. 49 (2012), No. 6, pp. 1251-1254

http://dx.doi.org/10.4134/BKMS.2012.49.6.1251

\title{
NOTE ON PURE-STRATEGY NASH EQUILIBRIA IN MATRIX GAMES
}

\author{
WEIDONG MA
}

\begin{abstract}
Pure-strategy Nash Equilibrium (NE) is one of the most important concepts in game theory. Tae-Hwan Yoon and O-Hun Kwon gave a "sufficient condition" for the existence of pure-strategy NEs in matrix games [5]. They also claimed that the condition was necessary for the existence of pure-strategy NEs in undominated matrix games. In this short note, we show that these claims are not true by giving two examples.
\end{abstract}

\section{Introduction}

In 1951, John Nash proved that every finite game has at least one mixed strategy Nash Equilibrium (NE) [2]. However, sometimes one may be interested in pure-strategy NE. Thus, a sufficient condition for the existence of purestrategy equilibria is very important. A two-player zero-sum game can be completely determined by its corresponding payoff matrix. It is, therefore, called a matrix game [4]. In such a game, a saddle point is essentially coincident with a pure-strategy NE [4]. In other words, the existence of saddle point is a sufficient and necessary condition for the existence of pure-strategy NE.

Tae-Hwan Yoon and O-Hun Kwon gave a "sufficient condition" for the existence of pure-strategy equilibria in matrix games [5]. They also claimed that the condition was necessary in undominated matrix games. However, we give two examples to show that such claims are not true.

\section{Preliminaries}

In this paper, we will adopt the same notations as used [5]. Let $\Gamma_{A}$ denote an $(m \times n)$ matrix game, and $A=\left(a_{i j}\right), a_{i j}>0$ be the payoff matrix of one of the two players. In addition, for a matrix game $\Gamma_{A}$, the strategy profile $(i, j)$ is

Received January 13, 2011.

2010 Mathematics Subject Classification. 91A05.

Key words and phrases. pure-strategy equilibrium, matrix game, saddle point.

This work was supported in part by the National Natural Science Foundation of China under Grant No. 11021161, 10928102 and Chinese Academy of Sciences under Grant No. kjcx-yw-s7. 
a saddle point [3] of $A$, if the corresponding element $a_{i j}$ of $A$ is simultaneously a minimum in its row and a maximum in its column, i.e.,

$$
a_{i j} \geq a_{k j}, \quad \forall k \text { and } a_{i j} \leq a_{i l}, \quad \forall l \text {. }
$$

A Nash equilibrium [1] is a set of strategies, one for each player, such that no player has incentive to unilaterally change his/her action, because no player can benefit by changing his/her strategy while the other players keep theirs unchanged.

\section{Two examples}

The following theorem gives a sufficient and necessary condition for the existence of pure-strategy NE.

Theorem 3.1. The strategy profile $(i, j)$ is a saddle point if and only if $(i, j)$ is a Nash equilibrium.

Proof. If $(i, j)$ is a Nash equilibrium, then by definition $a_{i j} \geq a_{k j}$ for any $k$, and $a_{i j} \leq a_{i l}$ for any $l$. Hence, $(i, j)$ is a saddle point.

Now suppose that $(i, j)$ is a saddle point, then $a_{i j}$ is maximum in its column hence the row player cannot increase his/her payoff given that column player has chosen column $j$. Similarly the column player cannot increase his/her payoff by changing his/her strategy given that the row player has chosen row $i$. Hence $(i, j)$ is a Nash equilibrium.

From the above theorem, we know that in order to find a pure-strategy NE, we only need to find a saddle point of the payoff matrix $A$. The authors of [5] claimed the following.

Claim 3.1 (Theorem 5 of [5]). For the existence of an equilibrium point in pure-strategies in $\Gamma_{A}$, it is sufficient that there exists a solution $\mathbf{x}_{0}$ in $\mathbb{R}^{m}$ of the system

$$
\left\{\begin{array}{c}
\mathbf{x} A \in \mathbb{R}_{-}^{n} \cup\left\{\mathbf{0}_{n}\right\}, \\
\mathbf{x} \cdot \mathbf{1}_{m}>0
\end{array}\right.
$$

and a solution $\mathbf{y}_{0}$ in $\mathbb{R}^{n}$ of the system

$$
\left\{\begin{array}{c}
A \mathbf{y} \in \mathbb{R}_{+}^{m} \cup\left\{\mathbf{0}_{m}\right\}, \\
\mathbf{y} \cdot \mathbf{1}_{n}<0 .
\end{array}\right.
$$

Claim 3.2 (Theorem 6 of [5]). For the existence of an equilibrium point in purestrategies in an undominated matrix game $\Gamma_{A}$, it is necessary and sufficient that there exists a solution $\mathbf{x}_{0}$ in $\mathbb{R}^{m}$ of the system (3.1) and a solution $\mathbf{y}_{0}$ in $\mathbb{R}^{n}$ of the system (3.2). 
The following two examples illustrate that both claims are incorrect. Consider two games $\Gamma_{A}$ and $\Gamma_{B}$, with following payoff matrices

$$
A=\left(\begin{array}{ccc}
3 & 0 & 1 \\
0 & 3 & 1 \\
1 & 1 & \frac{2}{3}
\end{array}\right) \quad \text { and } B=\left(\begin{array}{ccc}
2 & 0 & 1 \\
0 & 2 & 1 \\
1 & 1 & 1
\end{array}\right), \text { respectively }
$$

For game $\Gamma_{A}$, it is easy to check that $A$ has no saddle point, thus we know, from Theorem 3.1, that $\Gamma_{A}$ has no pure-strategy NE. On the other hand, $\mathbf{x}=$ $\left(-1,-1, \frac{5}{2}\right)$ and $\mathbf{y}=\left(1,1,-\frac{5}{2}\right)$ are solutions for system (3.1) and system (3.2), respectively, thus it follows from Claim 3.1 that $\Gamma_{A}$ has a pure-strategy NE, contracting Theorem 1. So Claim 3.1 is incorrect.

For game $\Gamma_{B}$, strategy profile $(3,3)$ is a saddle point, thus strategy profile $(3,3)$ is a pure-strategy $\mathrm{NE}$ of $\Gamma_{B}$. Moreover, $\Gamma_{B}$ is an undominated matrix game. Thus, it follows from Claim 3.2 that system (3.1) and system (3.2) both have solutions. However, for any $\mathbf{x}=\left(x_{1}, x_{2}, x_{3}\right)$, if $\mathbf{x}$ is a solution of system (3.1), we have $\mathbf{x} B \leq 0$ and $\mathbf{x} \cdot \mathbf{1}>0$, which imply that $x_{1}+x_{2}+x_{3} \leq 0$ and $x_{1}+x_{2}+x_{3}>0$ are both satisfied, a contradiction. Hence Claim 3.2 is also incorrect.

We also note that the authors of [5] assumed that $a_{i j}>0$ for any $i, j$, and in our above examples some elements in the matrices are 0 . In fact, we can get the same conclusions by modifying matrices $A$ and $B$ as follows.

$$
A=\left(\begin{array}{ccc}
3 & 3 \epsilon & 1+\epsilon \\
3 \epsilon & 3 & 1+\epsilon \\
1+\epsilon & 1+\epsilon & \frac{2}{3}(1+\epsilon)
\end{array}\right) \quad \text { and } \quad B=\left(\begin{array}{ccc}
2 & 2 \epsilon & 1+\epsilon \\
2 \epsilon & 2 & 1+\epsilon \\
1+\epsilon & 1+\epsilon & 1+\epsilon
\end{array}\right)
$$

where $\epsilon>0$ is a small positive constant.

One of the two mistakes in [5] appeared in the proof of Claim 3.1. The authors claimed: If the vector $\mathbf{1}_{m}$ at the point $\overline{\mathbf{x}}$ is not in the cone generated by column vectors $a^{j_{i}}, \overline{\mathbf{x}}$ is an extreme point of the feasible region of (7) on the $k$-axis for some $k$. This claim, however, dose not hold. For instance, in our game $\Gamma_{A}$, it is easy to check that $\overline{\mathbf{x}}=\left(\frac{1}{2}, \frac{1}{2}, 0\right)$, but it is not an extreme point.

The other mistake appeared in the proof of Claim 3.2. The authors claimed: Thus, since any strategy of player 1 is not dominated by another, the vector $\mathbf{1}_{m}$ at the point $\overline{\mathbf{x}}$ is not in the cone generated by column vectors. This claim, however, dose not hold either. For instance, in our game $\Gamma_{B}, \overline{\mathbf{x}}=(0,0,1)$, but $\mathbf{1}_{m} \in$ cone $\left\{a^{j}: 1 \leq j \leq 3\right\}$, since $a^{3}=(1,1,1)$.

\section{Conclusions}

In matrix games, the existence of saddle points is an sufficient and necessary condition for the existence of pure-strategy NEs. However, for other games, there is no such good condition. Thus, finding sufficient conditions for the existence of pure-strategy NEs in general games is of great importance in practice and needs to be further studied. 


\section{References}

[1] D. Fudenberg and J. Tirole, Game Theory, The MIT Press, 1992.

[2] J. Nash, Non-cooperative games, Ann. of Math. (2) 54 (1951), 289-295.

[3] G. Owen, Game Theory, Academic Press, 1982.

[4] J. H. Wang, The Theory of Games, TsingHua University Press, 1988.

[5] T. Yoon and O. Kwon, On pure-strategy equilibria in matrix games, Bull. Korean Math. Soc. 37 (2000), no. 2, 377-385.

Institute of Applied Mathematics AMSS

Chinese Academy of Sciences BeiJing 100190, P. R. China

E-mail address: mawd@amss.ac.cn 